\title{
Kernos
}

Revue internationale et pluridisciplinaire de religion grecque antique

$26 \mid 2013$

Varia

\section{Thierry Petit, Ædipe et le Chérubin. Les sphinx levantins, cypriotes et grecs comme gardiens d'immortalité}

\section{Anne-Françoise Jaccottet}

\section{(2) OpenEdition}

1 Journals

\section{Édition électronique}

URL : http://journals.openedition.org/kernos/2172

DOI : $10.4000 /$ kernos. 2172

ISSN : 2034-7871

Éditeur

Centre international d'étude de la religion grecque antique

\section{Édition imprimée}

Date de publication : 10 octobre 2013

Pagination : 431-435

ISSN : 0776-3824

\section{Référence électronique}

Anne-Françoise Jaccottet, «Thierry Petit, EEdipe et le Chérubin. Les sphinx levantins, cypriotes et grecs comme gardiens d'immortalité », Kernos [En ligne], 26 | 2013, mis en ligne le 10 octobre 2013, consulté le 02 mars 2021. URL : http://journals.openedition.org/kernos/2172 ; DOI : https://doi.org/ $10.4000 /$ kernos. 2172

Ce document a été généré automatiquement le 2 mars 2021.

Kernos 


\title{
Thierry Petit, Edipe et le Chérubin. Les sphinx levantins, cypriotes et grecs comme gardiens d'immortalité
}

\author{
Anne-Françoise Jaccottet
}

\section{RÉFÉRENCE}

Thierry Petit, ÆEdipe et le Chérubin. Les sphinx levantins, cypriotes et grecs comme gardiens d'immortalité, Fribourg / Göttingen, Academic Press Fribourg / Vandenhoeck \& Ruprecht, 2011. 1 vol. $16 \times 23,5 \mathrm{~cm}, 388$ p. (Orbis Biblicus et Orientalis, 248). ISBN : 978-3-7278-1692-5 (Academic Press) ou 978-3-525-54369-6 (Vandenhoeck \& Ruprecht).

Dès le titre, joliment accrocheur, le lecteur est averti qu'il entre dans un ouvrage programmatique. La figure du sphinx est analysée dans l'optique d'une continuité fonctionnelle qui se joue des ruptures culturelles, comme l'A. va se charger de le mettre en lumière au gré des trois parties qui constituent l'ouvrage: Le sphinx au Levant: le chérubin (19-56); Le sphinx à Chypre (57-95); Le sphinx grec (96-242) ${ }^{1}$. Édifice soigneusement construit, parcours minutieusement préparé qui prend le lecteur par la main pour l'emmener, dans une composition annulaire, du célèbre médaillon de la coupe attique du Vatican ${ }^{2}$ présentant le face à face d'ÆEdipe et de la Sphinx ${ }^{3}$ perchée sur une colonne, à cette même représentation, après avoir parcouru, dans une sorte de voyage initiatique, le Levant, Chypre et le monde égéen à la découverte du sens symbolique, fonctionnel, univoque et ubiquitaire de la figure du sphinx. Autant dire que la masse des documents et des contextes convoqués, et la richesse des références bibliographiques invoquées rendent impossible tout compte rendu détaillé ou exhaustif de l'ouvrage. Tout au plus, après un résumé de la thèse soutenue, sera-t-il ici question 
des lignes de méthode, des présupposés et des principes scientifiques à la base de cette étude.

Partant du Levant comme première terre d'accueil du sphinx d'origine égyptienne, l'A. fait dialoguer les images et/ou textes égyptiens, babyloniens, palestiniens pour arriver à la conclusion que les sphinx - ou chérubins dans la tradition biblique -, le plus souvent en paire, sont les mandataires («hypostases») de la divinité principale (féminine sauf dans le cas de la divinité judéenne) et sont commis à la garde et à la vénération de l'Arbre de la Vie, métaphore de l'immortalité; c'est dans ce sens qu'il convient de comprendre les représentations de paires de sphinx héraldiques de part et d'autre d'une figure végétale - qui peut prendre des formes très diverses selon le stade de stylisation - dans laquelle l'A. reconnaît l'Arbre de la Vie que les sphinx protègent ou honorent, en particulier en levant une de leur pattes antérieures en direction de "l'Arbre »; ils ont donc comme rôle particulier "d'accorder, en tant que mandataires de la divinité, l'accès à l'Arbre de la (Sur)vie au mortel qui s'en est montré digne, et, en priorité, au Roi, incarnation terrestre du Dieu royal, auquel il s'identifie post mortem » (53). Se dégage dès cette première partie un groupe ternaire, Arbre de la Vie Déesse - sphinx, auquel s'adjoint encore la figure du Roi-Dieu, lié de façon particulière à la Déesse qui lui accorde en priorité l'immortalité.

3 C'est avec ce modèle interprétatif comme grille de lecture que l'A. aborde les deux autres volets de son parcours: les titres identiques donnés aux sous-chapitres des différentes parties soulignent dès la table des matières l'analogie de la démarche interprétative entre Levant Chypre et monde grec. Chypre tout d'abord, "plaque tournante » ou « creuset » (2), dont la particularité est de n'offrir aucun appui textuel à l'interprétation. Ce sont donc les images et elles seules qui formeront la matière de l'analyse. Passant en revue un nombre considérable de documents figurés, l'A. isole et retrouve les motifs mis en lumière dans le chapitre précédent pour arriver à la conclusion que «l'association des trois motifs [...] correspond à celle que l'on a pu observer en Orient, et tout porte à croire que sa signification est identique dans l'île " (86); la figure du Roi-Dieu, quatrième élément du système mis en place, est plutôt ici une figure du Dieu-Roi, inversion qui ne nuit pas au parallélisme parfait que construit l'A. entre Levant et Chypre, tant pour la figure du sphinx que pour son sens eschatologique, en lui-même et en combinaison avec les autres éléments identifiés.

4 Après cette mise en bouche, reste le plat principal (près de 150 pages) : le sphinx grec. Le scénario (pour reprendre les mots de l'A.) mis en place pour l'Orient et Chypre s'applique-t-il au monde grec? "Nous aidant de ce que nous savons du sphinx en Orient et à Chypre, il sera au moins possible de vérifier, sur une base au départ strictement figurative, si le sphinx hellénique peut revêtir une signification similaire à celle de son cousin Cypro-levantin. De là, on pourra revenir au texte et examiner si la signification ainsi supposée ne peut éclairer le sens même du mythe» (97). Passant par une brève histoire du sphinx dans le monde égéen, l'A. s'arrête plus longuement sur l'historiographie liée à l'hybride sur sol grec : occasion de stigmatiser, le plus souvent à juste titre, les blocages de la recherche $\mathrm{du} \mathrm{xx}^{\mathrm{e}} \mathrm{s}$. qui boude à l'excès la piste orientale, dans la foulée du miracle grec, et accorde au mythe d'ÆEdipe (et au texte en général) une place par trop écrasante. Partant donc des images, l'A. démonte, pour la figure du sphinx, les classifications antérieures en divers types, cherchant à prouver qu'en l'absence d'une typologie fiable susceptible de soutenir la diversité des fonctions jusque là proposée du sphinx grec, la seule solution logique est bien d'envisager une 
interprétation globale au niveau fonctionnel de cette figure ${ }^{4}$; et de proposer, à titre d'hypothèse de travail, la grille de lecture précédemment mise au point: "Nous testerons donc, pour le domaine hellénique, la validité du scénario qui rend le mieux compte de la fonction des sphinx levantins et cypriotes. Ainsi l'hybride grec sera envisagé successivement dans ses rapports avec les autres motifs qui constituent, en Orient et à Chypre, le même ensemble fonctionnel, à savoir l'Arbre de la Vie, la Déesse et le Dieu-Roi » (134).

C'est ainsi que l'A. part en quête des éléments de son groupe ternaire (voire quaternaire) : l'Arbre de la Vie, tout d'abord, qu'il reconnaît, depuis le Bronze récent jusqu'aux vases italiotes, en toute palmette, rosette, bouton de lotus ou tout élément végétal, quel que soit son stade d'abstraction et de stylisation, et dont la colonne ionique ou éolique avec son chapiteau à volutes est un symbole abrégé, simplifié, stylisé; la figure du sphinx, ensuite, reconnaissable aussi bien en trois dimensions, en sculpture, qu'en deux, sur les vases notamment et dont il détermine la fonction de "passeurs d'âme »; et enfin la Déesse qui, dans l'orbe grecque et par «la nature du panthéon», se démultiplie en déesses au pluriel, («les Grecs séparent donc ce qui ailleurs était uni » 195), Athéna, Héra, Artémis ou Aphrodite. Quant au Dieu-Roi, il se lit en filigrane derrière la figure des héros royaux (Héraclès, Persée, Thésée...), qui, aidés par une déesse dans leurs combats contre des monstres représentant les forces de la mort, acquièrent l'immortalité. Le scénario cypro-levantin fonctionne à nouveau, ce qu'il fallait démontrer...

6 Reste enfin à revenir à đEdipe, au mythe et au médaillon de la coupe du Vatican qui a ouvert cette étude et qui la clora. Les motifs que l'on considère, à tort il est vrai, comme secondaires sur ce tondo, comme la colonne ionique sur laquelle la Sphinx se tient et la palmette qui se développe derrière elle, donnent à la scène son vrai sens. Perché sur la colonne-Arbre de la Vie - dans un raccourci saisissant de la paire de sphinx protégeantadorant l'Arbre - la Sphinx, hypostase de la divinité, refuse aux Thébains l'accès à l'immortalité dont la colonne-Arbre est la métaphore. Ce refus est dû, comme dans l'interdiction d'accès à l'Arbre de la Vie de l'Eden au couple primordial (Genèse 3, 24) à une faute antérieure (ici celle de Laïos); le rôle du héros sera de rouvrir l'accès à l'immortalité, en rachetant la faute par sa soumission à une épreuve, celle de l'énigme. Dernière palmette interprétative, l'énigme de la Sphinx est mise en relation fonctionnelle avec les rituels d'initiation et en particulier avec les mots de passe que les défunts enterrés avec une lamelle "orphique " doivent, selon le texte même des lamelles (ou devrait-on préciser - de certaines d'entre elles seulement), prononcer devant les " gardiens » pour accéder à l'endroit privilégié qui leur est réservé dans l'audelà. Ces « gardiens » qui ouvrent les portes d'un au-delà privilégié et dionysiaque, que l'A. assimile à une immortalité, ne seraient-ils pas des sphinx ? En tant qu'assistants d'une grande déesse, en l'occurrence Perséphone, ils seraient tout naturellement les gardiens de l'immortalité dont les portes ne s'ouvrent que devant la bonne formule, comme devant la réponse à l'énigme. Preuve en sera pour l'A. la présence sur un vase italiote ${ }^{5}$ de deux sphinx supportant, chacun sur une colonne, le naiskos sous lequel apparaît Perséphone trônant en tant que reine des enfers.

7 Le médaillon de la coupe du Vatican, fruit des pérégrinations levantines, cypriotes et grecques de la figure «symbolique » du sphinx, a ainsi trouvé, au terme du parcours, son sens, initiatique, eschatologique, en tant que tout, aussi cohérent que le système qui nous est ici présenté par l'A. Le peintre (et Th. Petit dans la foulée ?) « a ainsi résolu 
le problème de 'l'infigurable dans la figure' qui étreint tout artiste aux prises avec le sacré » (240).

On saura gré à Th. Petit d'avoir osé remettre en question bien des a priori, bien des frontières, d'avoir voulu ouvrir le débat sur la figure du sphinx à une dimension interculturelle, d'avoir (re)donné aux représentations figurées une place centrale dans l'analyse. À ce titre, on soulignera les bénéfices incontestables de son analyse des «sphinx causeurs» et "sphinx ravisseurs» dans l'iconographie grecque: s'affranchissant du carcan analytique textuel et mythographique qui fait la part trop belle au modèle œdipéen, il démontre de façon convaincante qu'un sphinx en présence d'interlocuteurs ou enlevant une "victime " n'est pas forcément et a priori la Sphinx de la légende thébaine, mais peut-être bien un "passeur d'âmes » (126 et 176-182). On regrettera toutefois que cette libération d'un modèle interprétatif, que les postulats clairs et fondés énoncés dans les "principes herméneutiques" introductifs (10-18) auraient amplement suffi à justifier, ne se fasse en fin de compte que sous la pression d'un autre modèle, celui que l'A. bâtit et autour duquel il construit et conduit toute son analyse.

Ce cas de figure permet de comprendre les mécanismes du raisonnement et de l'analyse. Testant les diverses catégories iconographiques de sphinx grecs sur la base desquelles la critique a forgé l'image variée voire hétéroclite des fonctions de l'hybride, l'A. passe en revue huit types, ayant précisé que "seule une typologie strictement iconographique » (120) serait pertinente. Montrer les zones de recoupement entre ces divers types lui permet d'affirmer: «Puisque les cloisons entre les catégories typologiques que nous venons d'établir présentent des failles manifestes, de béantes brèches même, il faut peut-être se résoudre à considérer que même la simple typologie iconographie proposée ci-dessus, que nous avons pourtant tenté de déterminer sur des critères discrets et pertinents, seule garantie pour en établir la validité, ne permet pas de fonder une herméneutique. [...] Puisque nous avons échoué à prouver, sur une base exclusivement iconographique, l'existence de types distincts et irréductibles, il faut se résoudre à chercher une interprétation globale qui permettrait d'unifier fonctionnellement les divers avatars de l'hybride égéen" (134). Syllogisme parfait. Déduction logique. Le modèle unique et fonctionnel s'impose par le raisonnement objectif et factuel. Sauf que les types pris comme référence à cette analyse ne sont pas homogènes dans leur nature: des types formels et strictement iconographiques, comme le sphinx « héraldique ", "causeur » ou "ravisseur ", renvoyant à un schéma que l'on peut isoler graphiquement, voisinent avec des types fonctionnels, comme le sphinx « funéraire ", ou " héroïque ", ne répondant pas à une forme mais à un contenu thématique, à un contexte de fonctionnement; sans compter encore des sous-types, comme les sphinx "causeurs à la colonne », ou les sphinx "ravisseurs à la colonne", élevés au rang de types à part entière. On ne s'étonnera pas dès lors que les « cloisons " entre ces divers types analysés ne soient pas étanches, un schéma formel pouvant fort bien - et même souvent nécessairement - se combiner à une fonction : quoi de plus naturel que des sphinx "héraldiques» soient aussi "funéraires»? La logique implacable qui impose le modèle interprétatif global et fonctionnel est ainsi basée sur des prémisses biaisées. Le bel agencement qui donne à l'ensemble de la démonstration, ici comme de façon générale dans l'ouvrage, son fort ancrage logique est plus d'une fois le fait d'approximations, de synecdoques, de prises en compte partielles voire partiales des faits aussi bien que des analyses antérieures auxquelles l'A. renvoie en guise de références bibliographiques. Pour passer de « la simple description archéo-graphique » à 
«l'herméneutique proprement archéo-logique » (105), l'A. exploite les données qui sont utiles à la construction de son système, laissant dans l'ombre - inconsciemment sans doute - celles qui n'y entrent pas, procédant plus par analogie que par véritable logique. L'usage récurrent et même systématique du terme de symbole - que l'A. utilise comme synonyme ou variante stylistique de la métaphore - est symptomatique de ce glissement discret de la forme au fond, du schéma à sa fonction et à son sens, qui compromet plus d'une fois l'analyse véritablement iconographique du sujet en faisant glisser dans un même champ sémantique des schémas pourtant clairement distincts formellement. L'iconographie antique est-elle vraiment et en premier chef une «cosa simbolica » (120) comme le revendique l'A.?

10 C'est ainsi également que l'emploi d'un même vocabulaire unifiant, appliqué à des formes différentes - comme l'usage systématique de la notion « biblique » d'Arbre de la Vie à tout élément représentatif d'ordre végétal - enclenche de facto une lecture dirigée du motif en en faisant un symbole, et embraye automatiquement une interprétation fonctionnelle. La figure du sphinx, par exemple, n'est jamais définie schématiquement (sauf peut-être allusivement p. 20), l'accord sur sa dénomination n'est jamais qu'implicite comme issu d'une évidence, et les variations de sa représentation - jusqu'à son passage du masculin au féminin - ne sont pas déterminantes dans l'optique de l'A. Dans une perspective comparatiste comme celle revendiquée ici, l'usage raisonné et fondé d'un vocabulaire qui aura fait au préalable l'objet d'un accord de principe, même théorique, est pourtant bien la base et la clef de voûte de toute analyse scientifique.

11 On regrettera ainsi que 'l'acte de foi' de l'A. l'engage dans une construction à maintes reprises forcée et, de notre point de vue, biaisée de la question. Regret d'autant plus vif que plusieurs aspects ponctuels de la démonstration méritent la réflexion et sont susceptibles de nourrir de façon novatrice d'autres recherches, notamment par l'ouverture proposée.

12 Au travers des pérégrinations de la figure du sphinx, c'est toute la question de la dynamique des cultures en contacts qui est ici posée : un schéma iconographique, une forme voyage-t-elle avec son sens? Si la réponse par trop univoque de l'A. n'a pas su nous convaincre, espérons que cet ouvrage foisonnant et aux références larges et riches suscitera d'autres approches, d'autres réponses, partielles, contextualisées et nuancées.

\section{NOTES}

1. Ces trois parties se développent après une brève «introduction » (1-4) qui pose le cadre de la recherche face à la fascination exercée par la figure du sphinx et son secret, non encore percé, un "préambule» (5-9) qui présente la coupe du Vatican (ci-dessous n. 2) et la focalisation abusive des exégètes sur les deux figures principales au détriment des motifs secondaires, et des «principes herméneutiques» (10-18), credo méthodologique basé autant sur E. Panofski que sur D. Arasse, dans un élargissement de perspective que nous ne pouvons que saluer. L'ouvrage est complété d'un bref épilogue sur le devenir du sphinx aux époques moderne voire contemporaine 
(243-245), d'une riche bibliographie, de deux index (général et textes cités) et de 191 illustrations noir-blanc, dont on soulignera la diversité, tout en regrettant, pour de nombreux exemples, la très mauvaise qualité de numérisation ou de résolution de l'image.

2. Coupe attique à figures rouges (médaillon intérieur), Musée du Vatican 16.541, Peintre d'CEdipe $A R V^{2}$ 451,1. Figure 1 dans l'ouvrage.

3. L'A. définit ainsi l'usage qu'il fait du masculin, du féminin et même du neutre pour la figure du sphinx : «On lui conservera l'article masculin, qui doit être entendu comme un neutre (genre qui manque grammaticalement en français); en effet, il se trouve - rarement il est vrai - des sphinx masculins en Grèce. Comme le présent essai entend s'appuyer tout d'abord sur l'imagerie, la présence de ces spécimens masculins justifie le parti-pris. On ne conservera la forme féminine que pour traiter du monstre thébain, seul sphinx à être cité par les textes littéraires et qui, en outre, par sa singularité, mérite la majuscule : la Sphinx » (102).

4. Cf. développement infra.

5. Cratère à volutes apulien, Karslruhe, Badisches Landesmuseum B4. Fig. 160 dans l'ouvrage.

\section{AUTEURS}

\section{ANNE-FRANÇOISE JACCOTTET}

Université de Genève 